\title{
Positive association between baseline brachial-ankle pulse wave velocity and the risk of new-onset diabetes in hypertensive patients
}

Yuanyuan Zhang ${ }^{1}$, Panpan He ${ }^{1}$, Youbao Li ${ }^{1}$, Yan Zhang ${ }^{2}$, Jianping Li ${ }^{2}$, Min Liang ${ }^{1}$, Guobao Wang ${ }^{1}$, Genfu Tang ${ }^{3}$,

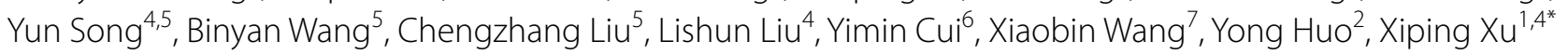
and Xianhui Qin ${ }^{1 *}$ (D)

\begin{abstract}
Background: There is no clearly defined temporal relationship between arterial stiffness and diabetes. We aimed to investigate the prospective association between baseline brachial-ankle pulse wave velocity (baPWV) and the risk of new-onset diabetes during follow-up, and examined whether there were effect modifiers, in hypertensive patients.

Methods: We included 2429 hypertensive patients with all the pertinent data but without diabetes at the baseline, who were part of the China Stroke Primary Prevention Trial (CSPPT), a randomized, double-blind, actively controlled trial conducted in 32 communities in Anhui and Jiangsu provinces in China. The primary outcome was new-onset diabetes, defined as physician-diagnosed diabetes or use of glucose-lowering drugs during follow-up, or fasting glucose $(\mathrm{FG}) \geq 126.0 \mathrm{mg} / \mathrm{dL}$ at the exit visit.

Results: During a median follow-up duration of 4.5 years, 287 (11.8\%) participants developed diabetes. There was a significant positive association between baseline baPWV and the risk of new-onset diabetes (per SD increment; OR, 1.33; $95 \% \mathrm{Cl} 1.13,1.56)$. Consistently, when baPWV was assessed as quartiles, a significantly higher risk of new-onset diabetes was found in participants in quartiles $2-4$ ( $\geq 15.9 \mathrm{~m} / \mathrm{s} ; \mathrm{OR}, 1.80 ; 95 \% \mathrm{Cl} 1.22,2.65)$ compared with those in quartile $1(<15.9 \mathrm{~m} / \mathrm{s})$. The positive association was consistent in participants with (per SD increment; OR, 1.29; 95\% Cl 1.06, 1.56) or without (per SD increment; $\mathrm{OR}, 1.40 ; 95 \% \mathrm{Cl} 1.15,1.71$ ) impaired fasting glucose (IFG, $\mathrm{FG} \geq 100.8$ and $<126.0 \mathrm{mg} / \mathrm{dL}$, -interaction $=0.486$ ).
\end{abstract}

Conclusions: In this sample of hypertensive patients, we found a significant positive association between baseline baPWV and the risk of new-onset diabetes.

Clinical trial registration Trial registration: NCT00794885 (clinicaltrials.gov). Retrospectively registered November 20, 2008

Keywords: Brachial-ankle pulse wave velocity, New-onset diabetes, Fasting glucose, Hypertensive patients

\footnotetext{
*Correspondence: xipingxu126@126.com; pharmaqin@126.com

${ }^{1}$ National Clinical Research Center for Kidney Disease; The State Key Laboratory for Organ Failure Research; Division of Nephrology, Nanfang

Hospital, Southern Medical University, Guangzhou 510515, China

Full list of author information is available at the end of the article
} 


\section{Background}

Diabetes is a worldwide public health problem [1]. Diabetes has been reported to be an important risk factor for cardiovascular disease (CVD) [2] and chronic kidney diseases [3]. Therefore, early identification of individuals at high-risk of developing diabetes is of clinical importance, for early risk assessment and intervention can help prevent the onset and slow the progress of diabetes and its related CVD complications.

Arterial stiffness increases with advancing age and has been regarded as an important risk factor for age-related morbidity and mortality $[4,5]$. Currently, because of its reproducibility and simplicity $[6,7]$, brachia-ankle pulse wave velocity (baPWV) is a widely used method for assessing arterial stiffness in studies of large sample sizes [7]. baPWV was found to be related to increased risk of stroke [8], total cardiovascular events and all-cause mortality [9], and was shown to positively associated with hypertension [10] and the presence of coronary calcium [11] or left ventricular mass [12]. Nevertheless, there are limited studies on baPWV and diabetes. Only some cross-sectional studies [13-15] reported that baPWV was related to the prevalence of diabetes.

Of note, we have previously demonstrated that higher baPWV decreased the antihypertensive effect of antihypertensive treatment in hypertensive adults [16]. Another study showed that uncontrolled blood pressure (BP) was associated with increased risk of incident diabetes [17]. These results raise the possibility that there is a link between baPWV and incident diabetes, but few studies have been conducted to delineate their temporal and causal relationships [18].

To address the aforementioned gap, the current study aimed to evaluate the prospective association between baseline baPWV and new-onset diabetes during the longitudinal follow-up, and to examine any possible effect modifiers among hypertensive patients, using data from the China Stroke Primary Prevention Trial (CSPPT) [19, 20].

\section{Methods}

\section{Study participants and design}

All participants were part of the CSPPT (clinicaltrials. gov identifier: NCT00794885). Detailed methods and major findings of the CSPPT trial have been described previously [19-22]. Briefly, the CSPPT was a large, community-based, multi-site, randomized, double-blind, and actively controlled trial with a total of 20,702 participants in China. Eligible participants were men and women aged 45 to 75 years old who had hypertension, defined as seated, resting systolic $\mathrm{BP}(\mathrm{SBP}) \geq 140 \mathrm{mmHg}$ or diastolic $\mathrm{BP}(\mathrm{DBP}) \geq 90 \mathrm{mmHg}$ at both the screening and the recruitment visit, or who were on antihypertensive medications. The major exclusion criteria included history of physician-diagnosed stroke, myocardial infarction, heart failure, post-coronary revascularization, and/or congenital heart disease.

The present study is a post hoc analysis of the CSPPT on 3532 subjects with baPWV measurements at baseline. Of those, 2429 participants without peripheral artery occlusive disease (PAD) assessed by ankle-brachial index $(\mathrm{ABI}<0.9)[6,16]$, with complete data on fasting glucose at baseline, and with physician-diagnosed diabetes or use of glucose-lowering drugs during the follow-up or fasting glucose data at the exit visit, as well as who were free of diabetes (physician-diagnosed diabetes or using glucose-lowering drugs) and whose fasting glucose (FG) was $<126.0 \mathrm{mg} / \mathrm{dL}$ at baseline, were included in the final analysis (Additional file 1: Figure S1).

The parent study (the CSPPT) and the current study were approved by the Ethics Committee of the Institute of Biomedicine, Anhui Medical University, Hefei, China (Federalwide Assurance Number 00001263). All participants provided written informed consent.

\section{Intervention and follow-up}

Eligible participants were randomly assigned, in a 1:1 ratio, to one of two treatment groups: a daily oral dose of one tablet containing $10 \mathrm{mg}$ enalapril and $0.8 \mathrm{mg}$ folic acid (the enalapril-folic acid group), or a daily oral dose of one tablet containing $10 \mathrm{mg}$ enalapril only (the enalapril group). Participants were followed up every 3 months. During each follow-up visit, blood pressure was measured; study drug compliance, concomitant medication use, adverse events and possible endpoint events were documented by trained research staff and physicians. The study drug compliance was calculated as the percentage of days taking the study drugs during the trial.

\section{Data collection procedures}

Baseline data collection was conducted by trained research staff according to a standard operating procedure. Each participant was interviewed using a standardized questionnaire designed specifically for this study. The question about socioeconomic status was phrased as follows, "How does your standard of living compare to others?" and a choice of three responses: bad, medium, and good was provided. The question about physical activity was phrased as follows, "How do you describe your daily physical activity level?" and a choice of three responses: low, moderate, and high was provided [23-25]. 


\section{Laboratory assays}

Serum fasting glucose (FG), lipids, and creatinine levels were measured using automatic clinical analyzers (Beckman Coulter) at the core laboratory of the National Clinical Research Center for Kidney Disease, Nanfang Hospital, Guangzhou, China. Serum folate was measured at baseline by a commercial laboratory using a chemiluminescent immunoassay (New Industrial).

\section{baPWV measurements}

baPWV, calculated as the ratio of transmission distance from the brachium to the ankle divided by the transit time, was used in this study. Participants were asked to remain in the supine position for at least $5 \mathrm{~min}$ after which baseline baPWV was measured using an automatic waveform analyzer (form PWV/ABI, BP-203RPE; Omron-Colin, Japan) according to published guidelines. The details describing the method of obtaining baPWV measurements are published elsewhere $[8,16]$. In brief, two, bilateral readings of baPWV measurements were simultaneously taken and the maximum reading from each side was used for the analysis.

\section{Study outcomes}

The primary study outcome was new-onset diabetes, defined as physician-diagnosed diabetes, or use of glucose-lowering drugs during follow-up, or new onset $\mathrm{FG} \geq 126.0 \mathrm{mg} / \mathrm{dL}$ at the exit visit.

The secondary study outcomes include: (1) physiciandiagnosed diabetes, or use of glucose-lowering drugs during follow-up; (2) the change in FG, calculated as FG at the exit visit minus that at baseline. The analysis of change in FG included subjects without physician-diagnosed diabetes, or use of glucose-lowering drugs during the follow-up.

\section{Statistical analyses}

Baseline characteristics are presented as mean \pm standard deviation (SD) for continuous variables and proportions for categorical variables. Differences in baseline characteristics by baPWV quartiles were compared using ANOVA tests, or Chi-square tests, accordingly. The relationship of baPWV quartiles $(<15.9,15.9-<17.9$, $17.9-<20.7$, and $\geq 20.7 \mathrm{~m} / \mathrm{s}$ ) with new-onset diabetes (primary outcome), physician-diagnosed diabetes or use of glucose-lowering drugs during follow-up, and change in FG (secondary outcomes) were evaluated using multivariable logistic regression models, Cox proportional hazard regression models and generalized linear regression models, respectively, without and with adjustment for age, sex, study center, study treatment group, body mass index (BMI), heart rate, smoking, systolic blood pressure (SBP), fasting glucose, total cholesterol (TC), creatinine, and folate at baseline, as well as time-averaged SBP during the treatment period. As additional exploratory analyses, possible modifications on the association between baPWV and new-onset diabetes were also evaluated by stratified analyses and interaction testing.

A two-tailed $P<0.05$ was considered to be statistically significant in all analyses. $\mathrm{R}$ software (version 3.4.3, http://www.R-project.org) were used for all statistical analyses.

\section{Results}

Study participants and baseline characteristics

As illustrated in the flow chart (Additional file 1: Figure S1), a total of 2429 hypertensive participants of the CSPPT without diabetes at the baseline were included in the final analysis.

Baseline characteristics of the study participants by baPWV quartiles are shown in Table 1 . The mean age of the participants was 59.7 (SD, 7.4) years; 1049 were men (43.2\%). Mean baseline baPWV was 18.6 (SD, 3.7) $\mathrm{m} / \mathrm{s}$. baPWV levels were inversely associated with BMI and time averaged diastolic blood pressure (DBP) during the treatment period, and positively associated with age, heart rate, SBP, DBP, TC, fasting glucose, high-density-lipid cholesterol (HDL-C) at baseline, as well as time averaged SBP during the treatment period. Moreover, participants with higher baPWV seemed to have lower physical activity levels (Additional file 1: Table S1).

\section{Association between baseline baPWV and the study outcomes}

During a median follow-up duration of 4.5 years (IQR, 4.2-4.7 years), new-onset diabetes occurred in 287 (11.8\%) participants.

Overall, there was a significant positive association between baPWV and the risk of new-onset diabetes (Fig. 1). Per SD increment $(3.7 \mathrm{~m} / \mathrm{s})$, higher baPWV was associated with a $33 \%$ increase in the adjusted risk of new-onset diabetes (OR, 1.33; 95\% CI 1.13, 1.56) (Additional file 1: Table S2). When baPWV was assessed as quartiles, the adjusted odds ratios for participants in the second, third and fourth quartiles were 1.63 (95\% CI 1.06, 2.49), 1.87 (95\% CI 1.20, 2.91) and 2.48 (95\% CI $1.53,4.03)$, respectively, compared with those in quartile 1 ( $P$ for trend $<0.001$ ). A significantly higher risk of newonset diabetes was found in participants in quartiles 2-4 (adjusted OR, 1.80; 95\% CI 1.22, 2.65) compared with those in quartile 1 (Fig. 2, Additional file 1: Table S2).

Consistently, per each SD increment $(3.7 \mathrm{~m} / \mathrm{s})$, higher baPWV was associated with a $61 \%$ increase in the adjusted risk of physician-diagnosed diabetes or use of glucose-lowering drugs during follow-up (HR, 1.61; 95\% 
Table 1 Baseline characteristics of the study population by brachial-ankle pulse wave velocity (baPWV) quartiles

\begin{tabular}{|c|c|c|c|c|c|c|}
\hline \multirow[t]{2}{*}{ Variables } & \multirow[t]{2}{*}{ Total } & \multicolumn{4}{|l|}{ baPWV, m/s } & \multirow[t]{2}{*}{$P$ value } \\
\hline & & Quartile $1(<15.9)$ & Quartile $2(15.9-<17.9)$ & Quartile $3(17.9-<20.7)$ & Quartile 4 ( $\geq 20.7$ ) & \\
\hline N & 2429 & 607 & 605 & 606 & 611 & \\
\hline Age, years & $59.7 \pm 7.4$ & $55.2 \pm 6.3$ & $58.9 \pm 6.8$ & $60.9 \pm 6.9$ & $63.8 \pm 6.6$ & $<0.001$ \\
\hline Male, No. (\%) & $1049(43.2)$ & $273(45.0)$ & $267(44.1)$ & $252(41.6)$ & $257(42.1)$ & 0.581 \\
\hline Body mass index, $\mathrm{kg} / \mathrm{m}^{2}$ & $25.1 \pm 3.6$ & $25.7 \pm 3.5$ & $25.2 \pm 3.7$ & $24.8 \pm 3.5$ & $24.5 \pm 3.4$ & $<0.001$ \\
\hline Heart rate, bmp & $72.4 \pm 9.5$ & $70.1 \pm 8.5$ & $71.2 \pm 8.5$ & $72.3 \pm 9.2$ & $75.9 \pm 10.7$ & $<0.001$ \\
\hline baPWV, m/s & $18.6 \pm 3.7$ & $14.6 \pm 1.1$ & $16.9 \pm 0.6$ & $19.2 \pm 0.8$ & $23.7 \pm 2.8$ & $<0.001$ \\
\hline Current smoking, No. (\%) & $585(24.1)$ & $127(20.9)$ & $155(25.6)$ & $151(24.9)$ & $152(24.9)$ & 0.275 \\
\hline Enalapril group, No. (\%) & $1196(49.2)$ & $304(50.1)$ & $290(47.9)$ & $295(48.7)$ & $307(50.2)$ & 0.823 \\
\hline \multicolumn{6}{|c|}{ MTHFR C677T polymorphisms, No. (\%) } & 0.920 \\
\hline $\mathrm{CC}$ & $636(26.2)$ & $151(24.9)$ & $156(25.8)$ & $169(27.9)$ & $160(26.2)$ & \\
\hline $\mathrm{CT}$ & $1186(48.8)$ & $296(48.8)$ & $297(49.1)$ & $293(48.3)$ & $300(49.1)$ & \\
\hline $\mathrm{TT}$ & $607(25.0)$ & $160(26.4)$ & $152(25.1)$ & $144(23.8)$ & $151(24.7)$ & \\
\hline \multicolumn{7}{|l|}{$\mathrm{BP}, \mathrm{mmHg}$} \\
\hline Systolic BP at baseline & $166.5 \pm 20.2$ & $153.5 \pm 16.2$ & $163.4 \pm 16.1$ & $170.0 \pm 18.8$ & $179.1 \pm 20.0$ & $<0.001$ \\
\hline Diastolic BP at baseline & $94.8 \pm 11.7$ & $93.2 \pm 10.3$ & $94.1 \pm 11.3$ & $94.5 \pm 12.0$ & $97.4 \pm 12.6$ & $<0.001$ \\
\hline Time-averaged systolic $\mathrm{BP}^{\mathrm{a}}$ & $138.6 \pm 10.7$ & $133.7 \pm 9.0$ & $137.8 \pm 9.9$ & $139.2 \pm 10.1$ & $143.5 \pm 11.2$ & $<0.001$ \\
\hline Time-averaged diastolic BP & $83.3 \pm 7.4$ & $84.6 \pm 6.8$ & $83.6 \pm 7.4$ & $82.5 \pm 7.6$ & $82.4 \pm 7.4$ & $<0.001$ \\
\hline \multicolumn{7}{|l|}{ Laboratory results } \\
\hline $\mathrm{HDL}-\mathrm{C}, \mathrm{mmol} / \mathrm{L}$ & $1.3 \pm 0.3$ & $1.3 \pm 0.3$ & $1.3 \pm 0.4$ & $1.4 \pm 0.3$ & $1.4 \pm 0.4$ & $<0.001$ \\
\hline Total cholesterol, mmol/L & $5.4 \pm 1.1$ & $5.3 \pm 1.0$ & $5.4 \pm 1.1$ & $5.5 \pm 1.1$ & $5.6 \pm 1.2$ & $<0.001$ \\
\hline Triglycerides, mmol/L & $1.7 \pm 2.1$ & $1.6 \pm 1.0$ & $1.7 \pm 4.0$ & $1.6 \pm 0.8$ & $1.7 \pm 0.9$ & 0.606 \\
\hline Fasting glucose, mg/dL & $97.6 \pm 12.5$ & $97.0 \pm 11.9$ & $97.3 \pm 11.9$ & $97.1 \pm 12.7$ & $99.0 \pm 13.2$ & 0.016 \\
\hline Creatinine, $\mu \mathrm{mol} / \mathrm{L}$ & $65.3 \pm 15.2$ & $64.6 \pm 14.1$ & $65.2 \pm 14.1$ & $65.8 \pm 17.3$ & $65.7 \pm 15.2$ & 0.466 \\
\hline Folate, ng/mL & $8.2 \pm 3.6$ & $8.0 \pm 3.3$ & $8.2 \pm 3.5$ & $8.1 \pm 3.6$ & $8.4 \pm 3.9$ & 0.213 \\
\hline \multicolumn{7}{|l|}{ Medication use, No. (\%) } \\
\hline Antihypertensive drugs & $1152(47.4)$ & $292(48.1)$ & $276(45.6)$ & $294(48.5)$ & $290(47.5)$ & 0.755 \\
\hline Lipid lowering drugs & $18(0.7)$ & $6(1.0)$ & $7(1.2)$ & $1(0.2)$ & $4(0.7)$ & 0.193 \\
\hline Antiplatelet drugs & $67(2.8)$ & $17(2.8)$ & $12(2.0)$ & $14(2.3)$ & $24(3.9)$ & 0.177 \\
\hline
\end{tabular}

Variables are presented as mean \pm SD or $\mathrm{n}(\%)$

BP: blood pressure; HDL-C: high-density lipoprotein; MTHFR: methylenetetrahydrofolate reductase

a Time-averaged systolic BP: mean systolic BP during the treatment period

CI 1.05, 2.47) (Additional file 1: Figure S2, Table S3). Accordingly, a significantly higher increase of FG levels (FG at the exit visit minus that at baseline) was also found in participants in quartiles 4 (adjusted $\beta, 2.89 \mathrm{mg} / \mathrm{dL}$; 95\% CI 0.12, 5.66) compared with those in quartile 1 of the baPWV levels among participants without physiciandiagnosed diabetes, or use of glucose-lowering drugs during follow-up (Additional file 1: Figure S3, Table S4).

\section{Sensitivity analysis}

During the treatment period, participants with higher baPWV had a higher frequency in use of calcium channel blockers (CCB) or diuretics (Additional file 1: Table S5). However, further adjustment for two variables: the use of calcium channel blockers (CCB), and the use of diuretics during the treatment period, did not substantially change the association between baPWV and new-onset diabetes (Additional file 1: Table S6). Moreover, the similar results were also found in participants without the concomitant use of diuretics during the treatment period (Additional file 1: Table S7).

In the CSPPT, all participants used enalapril or enalapril- folic acid during the follow up. However, further adjustment for the study drug (enalapril or enalapril-folic acid) compliance during the trial did not substantially change the results (Additional file 1: Table S8). Furthermore, we have further adjusted for family history of diabetes, physical activity, alcohol consumption and socioeconomic status, the association between baPWV and new-onset diabetes also did not been changed materially 


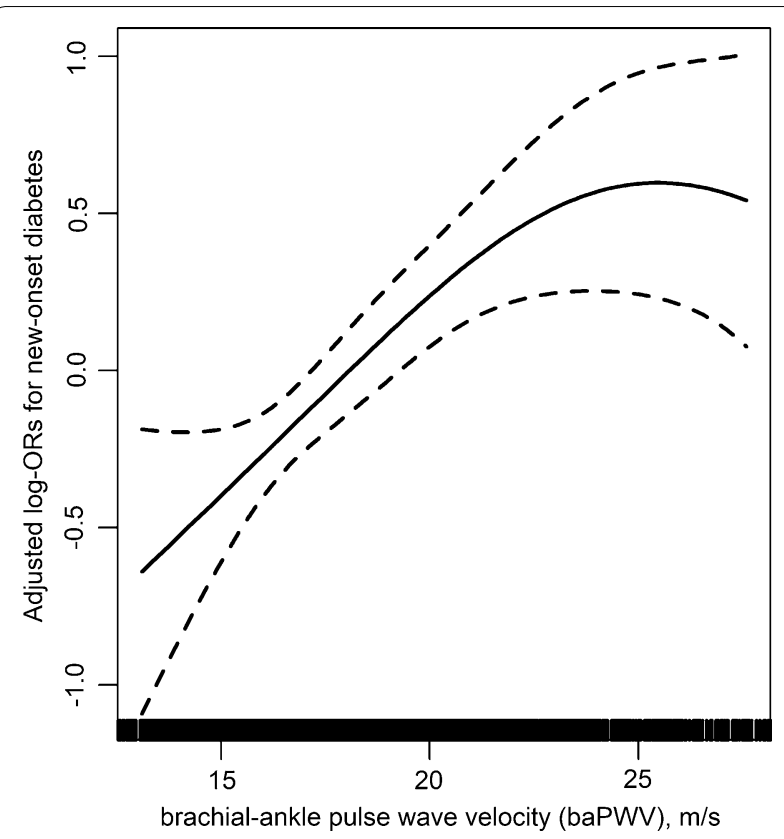

Fig. 1 Association between baseline brachial-ankle pulse wave velocity (baPWV) and new-onset diabetes during follow-up. *Adjusted for age, sex, study center, study treatment group, body mass index (BMI), heart rate, smoking, systolic blood pressure (SBP), fasting glucose (FG), total cholesterol (TC), creatinine, and folate at baseline, as well as time-averaged SBP during the treatment period
(Additional file 1: Table S9). More importantly, the similar results were also observed with further adjustment for the change in BMI (calculated as BMI at the exit visit minus that at baseline) (Additional file 1: Table S10).

In addition, we further explored the relationship of pulse pressure (PP) (both baseline PP and time-averaged PP during the treatment period) with new-onset diabetes (Additional file 1: Table S11). Overall, there was no significant association between baseline PP and newonset diabetes. However, there was a significant positive relationship of time-averaged PP with new-onset diabetes (quartile 3-4 vs. quartile 1 ; adjusted OR, 1.55 ; $95 \%$ CI 1.03, 2.33). Nevertheless, further adjustment for baseline PP and time-averaged PP during the treatment period did not substantially alter the positive association between baseline baPWV and the risk of new-onset diabetes (per SD increment; adjusted OR, 1.30; 95\% CI 1.11, 1.61) (Additional file 1: Table S12).

\section{Subgroup analyses by potential effect modifiers}

None of other variables, including sex (P-interaction $=0.275)$, age $(<60$ vs. $\geq 60$ years; $P$-interaction $=0.836), \quad$ BMI $\quad\left(<24, \quad 24-28, \quad \geq 28 \mathrm{~kg} / \mathrm{m}^{2}\right.$; $P$-interaction $=0.350$ ), treatment group (enalapril vs. enalapril + folic acid; $P$-interaction $=0.135)$, heart rate $[<72$ (median) vs. $\geq 72$ bmp; $P$-interaction $=0.420]$, SBP $(<160$ vs. $\geq 160 \mathrm{mmHg} ; P$-interaction $=0.747)$, TC $[<5.2$ vs. $\geq 5.2 \mathrm{mmol} / \mathrm{L} ; P$-interaction $=0.702]$, FG $(<100.8$ vs.

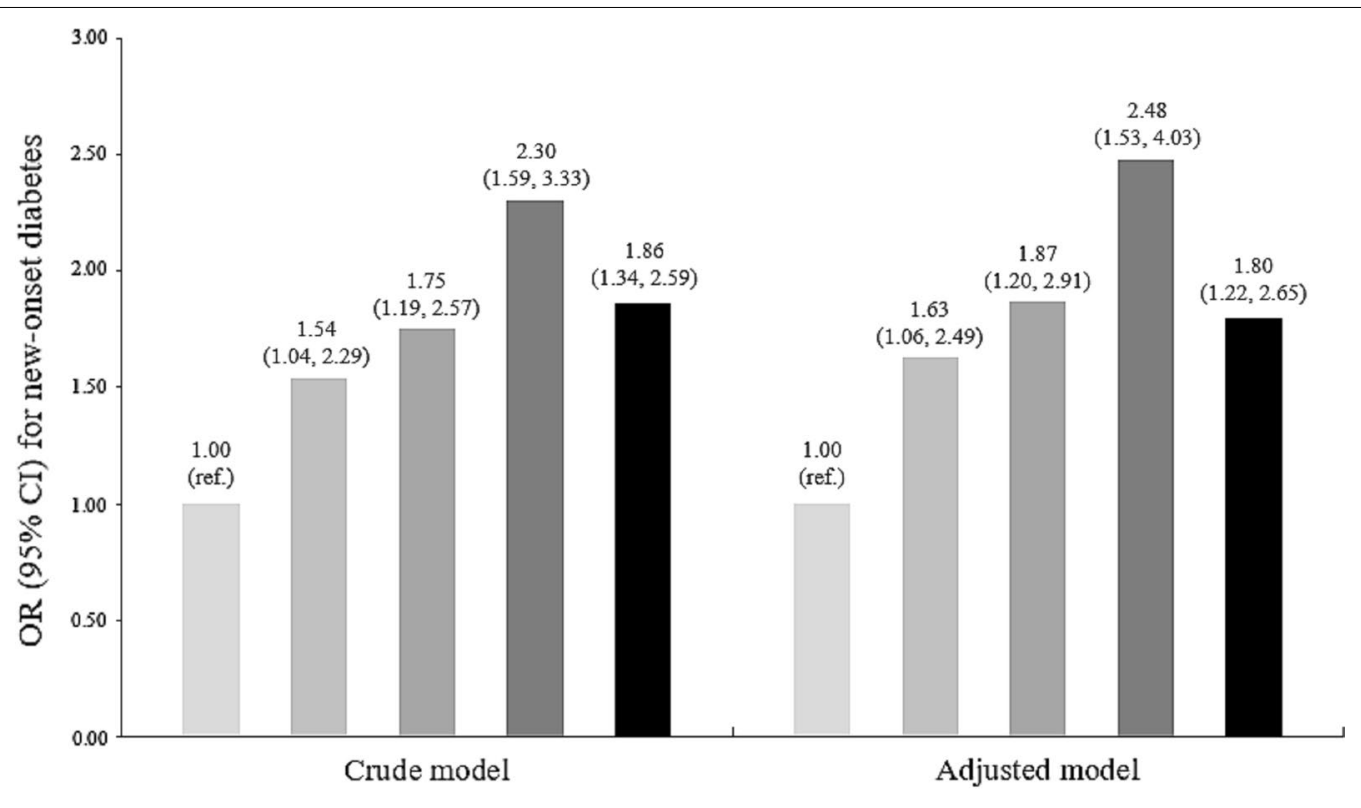

baPWV quartiles, $\mathrm{m} / \mathrm{s} \quad \mathrm{Q} 1(<15.9) \quad \square \mathrm{Q} 2(15.9-<17.9) \quad \square \mathrm{Q} 3(17.9-<20.7) \quad \square \mathrm{Q} 4(20.7) \quad \square \mathrm{Q} 2-4(\geq 15.9)$

Fig. 2 Risk of new-onset diabetes (expressed as OR and 95\% Cl) based on brachial-ankle pulse wave velocity (baPWV) quartiles. ${ }^{*}$ Adjusted for age, sex, study center, study treatment group, body mass index (BMI), heart rate, smoking, systolic blood pressure (SBP), fasting glucose (FG), total cholesterol (TC), creatinine, and folate at baseline, as well as time-averaged SBP during the treatment period 
$100.8-<126 \mathrm{mg} / \mathrm{mL} ; P$-interaction $=0.486)$, folate $[<7.7$ (median) vs. $\geq 7.7 \mathrm{ng} / \mathrm{mL} ; P$-interaction $=0.552]$ at baseline, and time-averaged SBP $(<140$ vs. $\geq 140 \mathrm{mmHg}$; $P$-interaction $=0.292$ ) during the treatment period, significantly modified the association between baPWV and new-onset diabetes (Fig. 3).

\section{Discussion}

To our knowledge, this is the first prospective study to demonstrate that baseline baPWV was positively associated with the risk of new-onset diabetes during a median follow-up of 4.5 years in hypertensive patients. The positive association was consistent across the strata for treatment group, sex, age, BMI, heart rate, SBP, TC, FG [with or without impaired fasting glucose (IFG: defined as a FG level $\geq 100.8$ and $<126.0 \mathrm{mg} / \mathrm{dL}$ )], and folate at baseline, as well as the time-averaged SBP during the follow-up period.

\section{Comparisons with previous studies}

Our results indicate that the traditional assumption that diabetes precedes arterial stiffness might need to be reconsidered. While some previous cross-sectional studies [13-15] have reported a positive association between baPWV and the prevalence of diabetes, the prospective association between diabetes and arterial stiffness is however, still inconclusive. de Oliveira Alvim et al. [26] found no significant difference in PWV progression after a 5-year follow-up in a subset of diabetics compared to non-diabetics. In contrast, Ferreira et al. [27] reported that better glycemic control, together with reductions in blood pressure and heart rate, was inversely associated with PWV changes. These results show that the association between arterial stiffness and diabetes is likely bidirectional. Diabetes might be in part a consequence of vascular impairment [28].

Our study findings are supported by previous studies. First, in a study on patients with untreated essential hypertension [29], those with higher pulse pressure (PP) exhibited impaired insulin secretion, increased post-challenge glucose concentrations and greater glucose spikes (PGS) during $75 \mathrm{~g}$ oral glucose tolerance testing. Our current study also found that there was a significant positive relationship of time-averaged PP with new-onset diabetes. Moreover, the positive association between baPWV and new-onset diabetes was independent of baseline PP

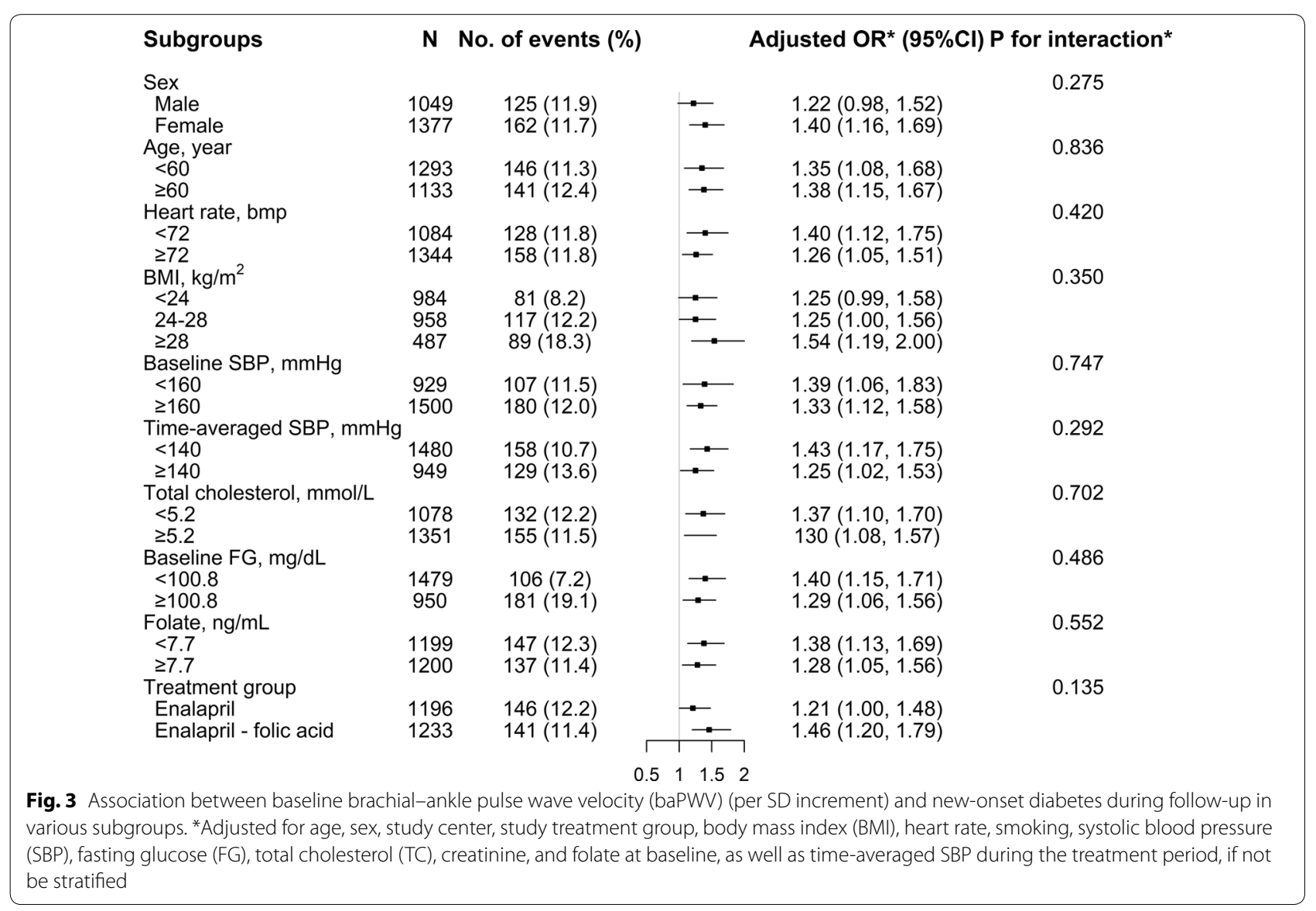


and time-averaged PP during the treatment period. These results suggested that baPWV may be a more accurate marker of arterial stiffness. Second, baPWV has been shown to be associated with endothelial dysfunction [30], hypertension [10], inflammation [31], triglyceride glucose (TyG) index [32], left ventricular hypertrophy (LVH) [12] and lower muscle tissue [33]. Previous studies have found that endothelial dysfunction [34], hypertension [35], inflammation [36], TyG index [37], and LVH [28, 38, 39] precede incident diabetes. Moreover, patients with type 2 diabetes and visceral fat accumulation usually had lower muscle quality [40]. Third, previous clinical trials in diabetic patients indicated that the control of hypertension and hypercholesterolemia is more effective than glucoselowering therapy in reducing cardiovascular events [41]. Fourth, angiotensin II receptor blocker (ARB) was found to inhibit the progression of arterial stiffness independent of blood pressure reduction [42]. Accordingly, ARB treatment significantly reduced the incidence of diabetes in previous studies $[43,44]$. Our results provide further evidence for the assumption that diabetes may be partly a disease of vascular origin. However, further studies are needed to verify this hypothesis.

\section{Possible mechanisms}

The exact mechanisms underlying the relationship of baPWV with new-onset diabetes remain to be elucidated. Some previously proposed potential mechanisms are outlined here. The propagation of increased pressure and flow pulsations to the pancreatic bed may lead to pancreatic dysfunction [18]. At the same time, arterial stiffness leads to increased arterial pulse pressure and pulsatile shear, resulting in endothelial dysfunction and metabolic dysregulation [30]. Endothelial dysfunction and impaired endothelium-dependent vasodilation may exacerbate insulin resistance by limiting the delivery of glucose to key target tissues [34]. More studies are needed to confirm our findings, and to further investigating the underlying mechanisms involved in this association.

\section{Limitations}

Our study has some limitations. First, arterial stiffness might represent the result of a number of risk factors, while the regression models were adjusted for a broad array of covariates, residual confounding from unmeasured factors cannot be excluded. Second, our present study was conducted in hypertensive participants, the generalizability of the results to adults without hypertension remains to be examined. Third, we did not measure glycosylated hemoglobin A1c or perform glucose tolerance tests. However, our definition of diabetes was similar to that of previous randomized trials $[45,46]$ or observational studies $[25,47,48]$. In addition, a glucose tolerance test is difficult to perform in practice, particularly in rural China. Finally, we did not have direct assessments of endothelial and $\beta$-cell function and insulin levels, which would have helped our understanding of the underlying pathophysiology and temporal sequence between baPWV and diabetes. Overall, our study served as hypothesis-generating; all findings need to be further investigated and confirmed in future studies.

\section{Conclusions}

In this sample of hypertensive patients, we found a significant positive association between baseline baPWV and the risk of new-onset diabetes during follow-up. If further confirmed, baPWV measurements along with other known risk factors could further help identify hypertensive patients at high-risk of developing diabetes. By targeting these high-risk patients and implementing early intensive multiple vascular risk factor control, we may help reduce their future diabetes risk.

\section{Supplementary information}

Supplementary information accompanies this paper at https://doi. org/10.1186/s12933-019-0915-0.

Additional file 1. Table S1. Baseline characteristics of the study population by brachial-ankle pulse wave velocity (baPWV) quartiles. Table S2. Association between baseline brachial-ankle pulse wave velocity (baPWV) and new-onset diabetes during follow-up. Table S3. Association between baseline brachial-ankle pulse wave velocity (baPWV) and physician-diagnosed diabetes or use of glucose-lowering drugs during follow-up. Table S4. Association between baseline brachial-ankle pulse wave velocity (baPWV) and the change in fasting glucose among subjects without physician-diagnosed diabetes, or use of glucose-lowering drugs during follow-up. Table S5. Concomitant use of medications during the treatment period by the quartiles of baseline brachial-ankle pulse wave velocity (baPWV). Table S6. Association between baseline brachial-ankle pulse wave velocity (baPWV) and new-onset diabetes during follow up, with further adjustment for the use of calcium channel blockers or diuretics during the treatment period. Table S7. Association between baseline brachial-ankle pulse wave velocity (baPWV) and new-onset diabetes during follow-up in participants without the use of diuretics during the treatment period. Table S8. Association between baseline brachial-ankle pulse wave velocity (baPWV) and new-onset diabetes during follow-up, with further adjustment for the study drug (enalapril or enalapril-folic acid) compliance during the trial. Table S9. Association between baseline brachial-ankle pulse wave velocity (baPWV) and new-onset diabetes during follow-up, with further adjustment for alcohol consumption, family history of diabetes, physical activity and socioeconomic status. Table S10. Association between baseline brachial-ankle pulse wave velocity (baPWV) and new-onset diabetes during follow-up, with further adjustment for change in BMI (calculated as BMI at the exit visit minus that at baseline). Table S11. Association between baseline pulse pressure (PP) (both baseline PP and time-averaged PP during the treatment period) and new-onset diabetes during follow-up. Table S12. Association between baseline brachial-ankle pulse wave velocity (baPWV) and new-onset diabetes during follow up, with adjustment for pulse pressure (PP) with or without systolic blood pressure (SBP). Figure S1. Flow chart of the study participants. Figure S2. Association between baseline brachial-ankle pulse wave velocity (baPWV) and physician-diagnosed diabetes or use of glucose-lowering drugs during follow-up. Figure S3. Association between baseline brachial-ankle pulse wave velocity (baPWV) and the 
change in fasting glucose (fasting glucose at the exit visit minus that at baseline) among subjects without physician-diagnosed diabetes, or use of glucose-lowering drugs during follow-up.

\section{Abbreviations}

ABI: ankle-brachial index; baPWV: brachial-ankle pulse wave velocity; BP: blood pressure; CCB: calcium channel blockers; CVD: cardiovascular disease; CSPPT: China Stroke Primary Prevention Trial; DBP: diastolic blood pressure; FG: fasting glucose; HDL-C: high-density lipoprotein; LVH: left ventricular hypertrophy; MTHFR: methylenetetrahydrofolate reductase; PP: pulse pressure; PAD: peripheral artery occlusive disease; SBP: systolic blood pressure.

\section{Acknowledgements}

We are grateful to the investigators and participants of the CSPPT, the parent study, who made this report possible.

\section{Authors' contributions}

YYZ, XPX and XHQ conceived and designed the study. YYZ, XHQ and CZL contributed to statistical analysis. YYZ, XHQ and XBW drafted the manuscript. All authors contributed to collect data and reviewed/edited the manuscript important intellectual content. All authors read and approved the final manuscript.

\section{Funding}

The study was supported by funding from the following: the National Key Research and Development Program [2016YFE0205400, 2018ZX09739, 2018ZX09301034003]; the Science and Technology Planning Project of Guangzhou, China [201707020010]; the Science, Technology and Innovation Committee of Shenzhen [JSGG20170412155639040, GJHS20170314114526143]; the Economic, Trade and Information Commission of Shenzhen Municipality [20170505161556110, 20170505160926390]; the National Natural Science Foundation of China [81730019]; Outstanding Youths Development Scheme of Nanfang Hospital, Southern Medical University [2017J009] and President Foundation of Nanfang Hospital, Southern Medical University [2017C007, 2018Z009].

\section{Availability of data and materials}

The data and study materials that support the findings of this study will be available from the corresponding authors upon request, after the request is submitted and formally reviewed and approved by the Ethics Committee of the Institute of Biomedicine, Anhui Medical University.

\section{Ethics approval and consent to participate}

The parent study (the CSPPT) and the current study were approved by the Ethics Committee of the Institute of Biomedicine, Anhui Medical University, Hefei, China (Federalwide Assurance Number 00001263). All participants provided written informed consent.

\section{Consent for publication}

Not applicable.

\section{Competing interests}

XPX reports grants from the National Key Research and Development Program [2016YFE0205400, 2018ZX09739, 2018ZX09301034003]; the Science and Technology Planning Project of Guangzhou, China [201707020010]; the Science, Technology and Innovation Committee of Shenzhen [JSGG20170412155639040, GJHS2017031411 4526143] and the Economic, Trade and Information Commission of Shenzhen Municipality [20170505161556110, 20170505160926390].XHQ reports grants from the National Natural Science Foundation of China [81730019] and Outstanding Youths Development Scheme of Nanfang Hospital, Southern Medical University [2017J009].YBL reports grants from the President Foundation of Nanfang Hospital, Southern Medical University [2017C007, 2018Z009].No other disclosures were reported.

\section{Author details}

${ }^{1}$ National Clinical Research Center for Kidney Disease; The State Key Laboratory for Organ Failure Research; Division of Nephrology, Nanfang Hospital, Southern Medical University, Guangzhou 510515, China. ${ }^{2}$ Department of Cardiology, Peking University First Hospital, Beijing 100034, China. ${ }^{3}$ Health Management College, Anhui Medical University, Hefei 230032, China. ${ }^{4}$ Beijing Advanced Innovation Center for Food Nutrition and Human Health, College of Food Science and Nutritional Engineering, China Agricultural University, Beijing 100083, China. ${ }^{5}$ Institute of Biomedicine, Anhui Medical University, Hefei 230032, China. ${ }^{6}$ Department of Pharmacy, Peking University First Hospital, Beijing 100034, China. ${ }^{7}$ Department of Population, Family and Reproductive Health, Johns Hopkins University Bloomberg School of Public Health, 615 N. Wolfe Street, E4132, Baltimore, MD 21205-2179, USA.

\section{Received: 5 August 2019 Accepted: 19 August 2019}

Published online: 28 August 2019

\section{References}

1. Guariguata L, Whiting DR, Hambleton I, Beagley J, Linnenkamp U, Shaw JE. Global estimates of diabetes prevalence for 2013 and projections for 2035. Diabetes Res Clin Pract. 2014;103:137-49.

2. Almdal T, Scharling $H$, Jensen JS, Vestergaard $H$. The independent effect of type 2 diabetes mellitus on ischemic heart disease, stroke, and death: a population-based study of 13000 men and women with 20 years of follow-up. Arch Intern Med. 2004;164:1422-6.

3. Zelnick LR, Weiss NS, Kestenbaum BR, Robinson-Cohen C, Heagerty PJ, Tuttle K, et al. Diabetes and CKD in the United States population, 2009-2014. Clin J Am Soc Nephrol. 2017;12:1984-90.

4. Kaess BM, Rong J, Larson MG, Hamburg NM, Vita JA, Levy D, et al. Aortic stiffness, blood pressure progression, and incident hypertension. JAMA. 2012;308:875-81

5. Cavalcante JL, Lima JA, Redheuil A, Al-Mallah MH. Aortic stiffness: current understanding and future directions. J Am Coll Cardiol. 2011;57:1511-22.

6. Munakata M. Brachial-ankle pulse wave velocity in the measurement of arterial stiffness: recent evidence and clinical applications. Curr Hypertens Rev. 2014;10:49-57.

7. Yamashina A, Tomiyama H, Takeda K, Tsuda H, Arai T, Hirose K, et al. Validity, reproducibility, and clinical significance of noninvasive brachial-ankle pulse wave velocity measurement. Hypertens Res. 2002;25:359-64.

8. Song Y, Xu B, Xu R, Tung R, Frank E, Tromble W, et al. Independent and joint effect of brachial-ankle pulse wave velocity and blood pressure control on incident stroke in hypertensive adults. Hypertension. 2016;68:46-53.

9. Vlachopoulos C, Aznaouridis K, Terentes-Printzios D, loakeimidis N, Stefanadis C. Prediction of cardiovascular events and all-cause mortality with brachial-ankle elasticity index: a systematic review and meta-analysis. Hypertension. 2012;60:556-62.

10. Tomiyama H, Komatsu S, Shiina K, Matsumoto C, Kimura K, Fujii M, et al. Effect of wave reflection and arterial stiffness on the risk of development of hypertension in Japanese men. J Am Heart Assoc. 2018;7:e008175.

11. Lee JY, Ryu S, Lee SH, Kim BJ, Kim BS, Kang JH, et al. Association between brachial-ankle pulse wave velocity and progression of coronary artery calcium: a prospective cohort study. Cardiovasc Diabetol. 2015;14:147.

12. Yu WC, Chuang SY, Lin YP, Chen CH. Brachial-ankle vs carotid-femoral pulse wave velocity as a determinant of cardiovascular structure and function. J Hum Hypertens. 2008;22:24-31.

13. Loehr LR, Meyer ML, Poon AK, Selvin E, Palta P, Tanaka H, et al. Prediabetes and diabetes are associated with arterial stiffness in older adults: the ARIC study. Am J Hypertens. 2016;29:1038-45.

14. Won KB, Chang HJ, Kim HC, Jeon K, Lee H, Shin S, et al. Differential impact of metabolic syndrome on subclinical atherosclerosis according to the presence of diabetes. Cardiovasc Diabetol. 2013;12:41.

15. Li C, Wu J, Yang Y, Shih C, Lu F, Chang C. Increased arterial stiffness in subjects with impaired glucose tolerance and newly diagnosed diabetes but not isolated impaired fasting glucose. J Clin Endocrinol Metab. 2012;97:E658-62.

16. Zheng M, Huo Y, Wang X, Xu X, Qin X, Tang G, et al. A prospective study on pulse wave velocity (PWV) and response to anti-hypertensive treatments: PWV determines BP control. Int J Cardiol. 2015;178:226-31.

17. Izzo R, de Simone G, Chinali M, laccarino G, Trimarco V, Rozza F, et al. Insufficient control of blood pressure and incident diabetes. Diabetes Care. 2009;32:845-50. 
18. Prenner SB, Chirinos JA. Arterial stiffness in diabetes mellitus. Atherosclerosis. 2015;238:370-9.

19. Huo Y, Li J, Qin X, Huang Y, Wang X, Gottesman RF, et al. Efficacy of folic acid therapy in primary prevention of stroke among adults with hypertension in China: the CSPPT randomized clinical trial. JAMA. 2015;313:1325-35.

20. Qin X, Li J, Zhang Y, Chen D, Wang B, He M, et al. Effect of folic acid supplementation on risk of new-onset diabetes in adults with hypertension in China: findings from the China Stroke Primary Prevention Trial (CSPPT). J Diabetes. 2016;8:286-94.

21. Zhao M, Wang X, He M, Qin X, Tang G, Huo Y, et al. Homocysteine and stroke risk: modifying effect of methylenetetrahydrofolate reductase C677T polymorphism and folic acid intervention. Stroke. 2017:48:1183-90.

22. Qin X, Li J, Spence JD, Zhang Y, Li Y, Wang X, et al. Folic acid therapy reduces the first stroke risk associated with hypercholesterolemia among hypertensive patients. Stroke. 2016;47:2805-12.

23. He M, Qin X, Cui Y, Cai Y, Sun L, Xu X, et al. Prevalence of unrecognized lower extremity peripheral arterial disease and the associated factors in Chinese hypertensive adults. Am J Cardiol. 2012;110:1692-8.

24. Qin X, Zhang Y, Cai Y, He M, Sun L, Fu J, et al. Prevalence of obesity, abdominal obesity and associated factors in hypertensive adults aged 45-75 years. Clin Nutr. 2013;32:361-7.

25. Qin X, Li J, Zhang Y, Ma W, Fan F, Wang B, et al. Prevalence and associated factors of diabetes and impaired fasting glucose in Chinese hypertensive adults aged 45 to 75 years. PLoS ONE. 2012;7:e42538.

26. de Oliveira Alvim R, Santos PCJL, Musso MM, de Sá Cunha R, Krieger JE, Mill JG, et al. Impact of diabetes mellitus on arterial stiffness in a representative sample of an urban Brazilian population. Diabetol Metab Syndr. 2013;5:45.

27. Ferreira MT, Leite NC, Cardoso CR, Salles GF. Correlates of aortic stiffness progression in patients with type 2 diabetes: importance of glycemic control: the Rio de Janeiro type 2 diabetes cohort study. Diabetes Care. 2015;38:897-904.

28. Izzo R, de Simone G, Trimarco V, Gerdts E, Giudice R, Vaccaro O, et al. Hypertensive target organ damage predicts incident diabetes mellitus. Eur Heart J. 2013;34:3419-26.

29. Anan F, Masaki T, Eto T, Fukunaga N, Iwao T, Kaneda K, et al. Postchallenge plasma glucose and glycemic spikes are associated with pulse pressure in patients with impaired glucose tolerance and essential hypertension. Hypertens Res. 2008;31:1565-71.

30. Petrie JR, GuzikTJ, Touyz RM. Diabetes, hypertension, and cardiovascular disease: clinical insights and vascular mechanisms. Can J Cardiol. 2018;34:575-84

31. Sun L, Ning C, Liu J, Yao T, Zhang L, Zhao L, et al. The association between cumulative C-reactive protein and brachial-ankle pulse wave velocity. Aging Clin Exp Res. 2019. https://doi.org/10.1007/s40520-019-01274-8.

32. Lee SB, Ahn CW, Lee BK, Kang S, Nam JS, You JH, et al. Association between triglyceride glucose index and arterial stiffness in Korean adults. Cardiovasc Diabetol. 2018;17:41.

33. Rodriguez AJ, Karim MN, Srikanth V, Ebeling PR, Scott D. Lower muscle tissue is associated with higher pulse wave velocity: a systematic review and meta-analysis of observational study data. Clin Exp Pharmacol Physiol. 2017:44:980-92.
34. Balletshofer BM, Rittig K, Enderle MD, Volk A, Maerker E, Jacob S, et al. Endothelial dysfunction is detectable in young normotensive first-degree relatives of subjects with type 2 diabetes in association with insulin resistance. Circulation. 2000;101:1780-4.

35. Conen D, Ridker PM, Mora S, Buring JE, Glynn RJ. Blood pressure and risk of developing type 2 diabetes mellitus: the Women's Health Study. Eur Heart J. 2007;28:2937-43.

36. Spranger J, Kroke A, Möhlig M, Hoffmann K, Bergmann MM, Ristow M, et al. Inflammatory cytokines and the risk to develop type 2 diabetes: results of the prospective population-based European Prospective Investigation into Cancer and Nutrition (EPIC)-Potsdam Study. Diabetes. 2003;52:812-7.

37. Lee DY, Lee ES, Kim JH, Park SE, Park CY, Oh KW, et al. Predictive value of triglyceride glucose index for the risk of incident diabetes: a 4-year retrospective longitudinal study. PLOS ONE. 2016;11:e0163465.

38. de Simone G, Wang W, Best LG, Yeh F, Izzo R, Mancusi C, et al. Target organ damage and incident type 2 diabetes mellitus: the Strong Heart Study. Cardiovasc Diabetol. 2017;16:64.

39. Yang $L$, Yang $L$, Zhang $Y, X i$ B. Prevalence of target organ damage in Chinese hypertensive children and adolescents. Front Pediatr. 2018;6:333.

40. Murai J, Nishizawa H, Otsuka A, Fukuda S, Tanaka Y, Nagao H, et al. Low muscle quality in Japanese type 2 diabetic patients with visceral fat accumulation. Cardiovasc Diabetol. 2018;17:112

41. Mannucci E, Dicembrini I, Lauria A, Pozzilli P. Is glucose control important for prevention of cardiovascular disease in diabetes? Diabetes Care. 2013;36(Suppl 2):S259-63.

42. Agata J, Nagahara D, Kinoshita S, Takagawa Y, Moniwa N, Yoshida D, et al. Angiotensin II receptor blocker prevents increased arterial stiffness in patients with essential hypertension. Circ J. 2004;68:1194-8.

43. NAVIGATOR Study Group, McMurray JJ, Holman RR, Haffner SM, Bethel MA, Holzhauer B, et al. Effect of valsartan on the incidence of diabetes and cardiovascular events. N Engl J Med. 2010;362:1477-90.

44. Scheen AJ. Renin-angiotensin system inhibition prevents type 2 diabetes mellitus. Part 1. A meta-analysis of randomised clinical trials. Diabetes Metab. 2004;30:487-96.

45. Stranges S, Marshall JR, Natarajan R, Donahue RP, Trevisan M, Combs GF, et al. Effects of long-term selenium supplementation on the incidence of type 2 diabetes: a randomized trial. Ann Intern Med. 2007;147:217-23.

46. Lippman SM, Klein EA, Goodman PJ, Lucia MS, Thompson IM, Ford LG, et al. Effect of selenium and vitamin E on risk of prostate cancer and other cancers: the Selenium and Vitamin E Cancer Prevention Trial (SELECT). JAMA. 2009:301:39-51.

47. Bleys J, Navas-Acien A, Guallar E. Serum selenium and diabetes in U.S. adults. Diabetes Care. 2007;30:829-34.

48. Laclaustra M, Navas-Acien A, Stranges S, Ordovas JM, Guallar E. Serum selenium concentrations and diabetes in U.S. adults: National Health and Nutrition Examination Survey (NHANES) 2003-2004. Environ Health Perspect. 2009;117:1409-13.

\section{Publisher's Note}

Springer Nature remains neutral with regard to jurisdictional claims in published maps and institutional affiliations.

Ready to submit your research? Choose BMC and benefit from

- fast, convenient online submission

- thorough peer review by experienced researchers in your field

- rapid publication on acceptance

- support for research data, including large and complex data types

- gold Open Access which fosters wider collaboration and increased citations

- maximum visibility for your research: over 100M website views per year

At BMC, research is always in progress.

Learn more biomedcentral.com/submissions 